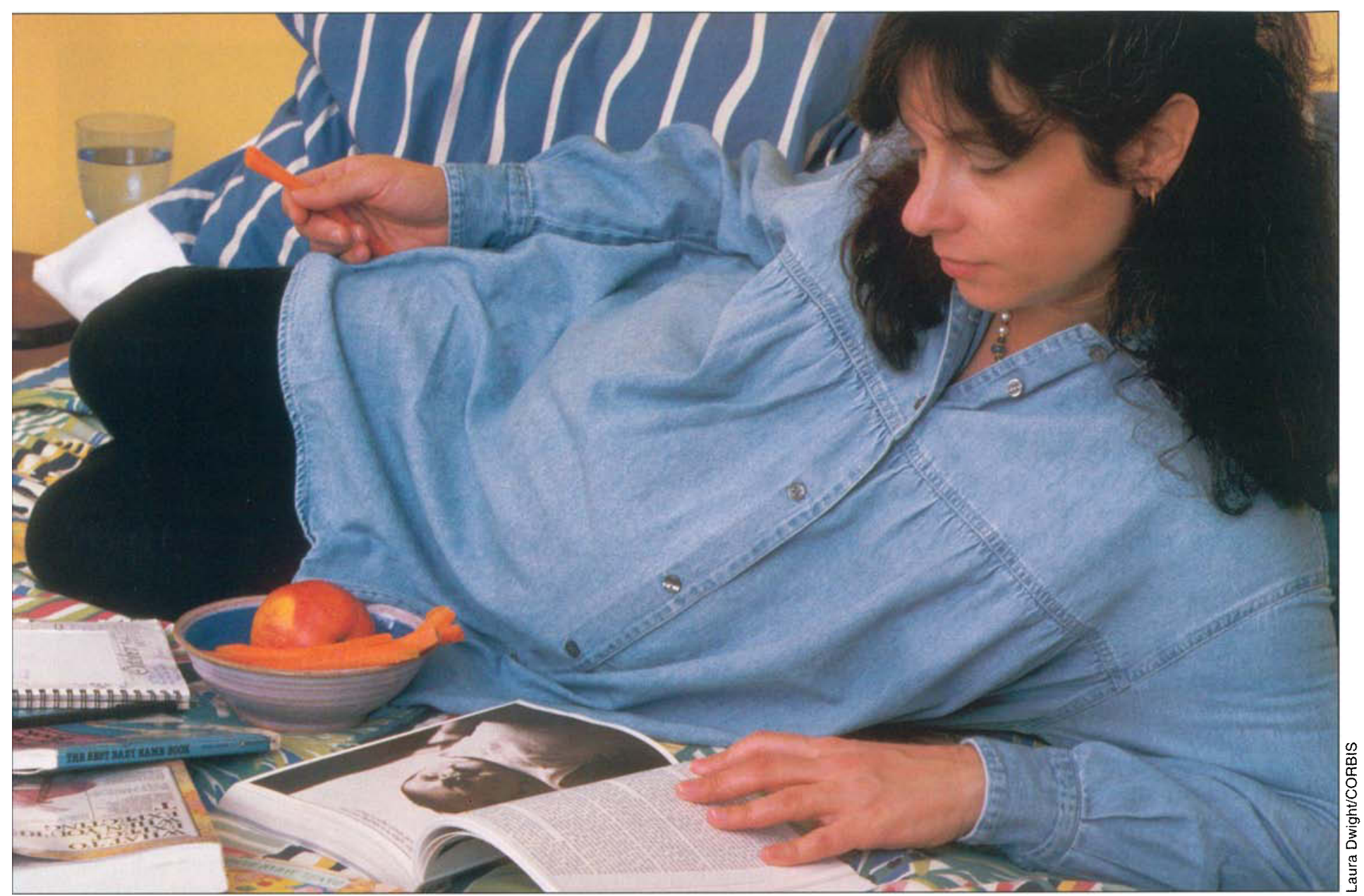

Suboptimal nutrition can play a significant role in pregnancy complications such as birth defects. A woman in her third trimester enjoys healthy snacks.

\title{
What are the best strategies for achieving optimal nutrition?
}

\author{
Carl L. Keen a Sheri Zidenberg-Cherr
}

Defining "optimal nutrition" has become more complicated with the growing awareness that a healthy diet may reduce the risk of cardiovascular disease, diabetes and other diseases as well as avert nutritional disorders. In contrast to the previous federal government's recommended dietary allowances (RDA), the newer dietary reference intake (DRI) committees are basing nutrient requirements on the contemporary concept of reducing disease risks as well as preventing nutrient deficiencies. Even when USDA food pyramid guidelines are followed, it can be difficult to meet current recommended intakes for essential nutrients. Rather than being rare, marginal nutritional deficiencies in the United States may in fact be quite common. For example, "suboptimal" maternal nutrition can be a significant factor underlying some pregnancy complications such as birth defects, yet a significant proportion of women of child-bearing age do not get sufficient nutrients from their diets. New programs are needed to improve the diets of pregnant women, as well as those of women who are planning pregnancy. In addition to recognized essential micronutrients, if protective dietary phytochemicals are identified, should we try to modify the content of foods, take dietary supplements or simply alter our diets? In the near future, a combination of the three may be the best strategy. Even if new food fortification and supplement policies are promulgated, they should complement, not replace, educational and economic programs designed to improve the public's overall diet. 
$\mathrm{D}$ uring the first half of the 20th century, the U.S. public's major dietary expectations were that food be plentiful and safe. Improving crop production was an important goal for agricultural scientists, while food scientists concentrated on ways to improve food storage, availability and safety. Nutrition scientists and publichealth officials expended considerable effort investigating the causes of major nutrition-related diseases and developing programs aimed at their eradication. The collective progress was tremendous; food production and safety were greatly enhanced, and several common nutritional disorders including rickets, pellagra and goiter were virtually eliminated through selective food fortification programs, such as bread and milk.

During the second half of the 20th century, crop production increased to levels that many would have thought unrealistic in the early 1900s. Food availability and safety also reached new heights. While hunger continues to be a problem for some segments of our society, the roots of this are more socioeconomic than due to the lack of plentiful food.

With respect to the nutritional sciences, the second half of the 20th century brought a growing awareness of the critical role that diet can play in the initial development and progression of a wide spectrum of diseases. The general public has learned that through good nutrition individuals can significantly reduce their risk for numerous diseases including hypertension, diabetes, macular degeneration, cardiovascular disease, osteoporosis, some types of cancer and Alzheimer's disease.

While many of the current dietary recommendations made by publichealth officials have been in place for several years (for example, consuming diets low in saturated fats, cholesterol and sodium), a number of new recommendations concerning the optimal intake of certain micronutrients have emerged. For example, during the past few years, pregnant women have been told that increasing their intake of folate can reduce the frequency and severity of birth defects. Likewise, di- ets rich in calcium and other micronutrients are widely believed to reduce the risk for osteoporosis. The public has also been informed that careful changes in diet (including food, beverages and dietary supplements), may even improve their physical and mental performance. Indeed, as we enter the 21st century, the majority of the U.S. public has accepted the concept that optimizing one's health potential will ultimately depend, in part, on the optimization of one's nutritional status. However, it is important to note that the research underlying many of the beliefs concerning nutrition and optimal health is still preliminary.

Underscoring this paradigm shift in nutrition is the current development of dietary reference intakes (DRIs) by the Food and Nutrition Board, which operates under the auspices of the National Academy of Sciences. In contrast to the previous recommended dietary allowances (RDAs), the new DRI committees do not determine nutrient requirements based solely on the prevention of nutrient deficiencies but rather on the contemporary concept of reducing disease risks using a specified indication of adequacy (Yates 1998). It is important to note that these new dietary recommendations are intended to complement rather than replace previous dietary guideline recommendations, such as those concerning dietary fat, cholesterol, sodium and fiber.

\section{Optimizing nutrition}

Is there substance to the new conventional wisdom on nutrition? And what can health professionals and the agricultural industry do to help ensure that a majority of the population achieves a condition approaching optimal nutrition status?

With respect to the first question, numerous epidemiological studies support the thesis that long-term dietary habits can influence one's susceptibility to many diseases. For example, several epidemiological studies have revealed that diets low in saturated fats and cholesterol, and rich in fruits and vegetables, can reduce the risk for coronary heart disease and
The antioxidant effects of vitamins $C$ and $E$ in buman plasma are prolonged in the presence of the phytochemical catechin, which is found in many foods including numerous fruits and vegetables, green tea, red wine and chocolate.

some cancers (Block et al. 1992; Hertog et al. 1995; Keli et al. 1996; Yochum et al. 1999). While most investigators accept this association, there is considerable debate over the relative roles that different dietary factors play in the initiation, progression or prevention of vascular disease. For example, there is a vigorous, ongoing debate over the identity of the components (often referred to as phytochemicals) present in fruits and vegetables that confer pre tective value.

Several research groups have proposed that the protective effects associated with fruits and vegetables can be attributed in part to their antioxidant content. Consequently, numerous long-term supplementation trials with several purified antioxidants (such as vitamin $E$, vitamin $C$ and beta-carotene) have been conducted. Regrettably, these trials have not yielded definitive positive results (HOPE 2000; ATBC 1994; Carr and Frei 1999). Multiple explanations can be given for why these trials have not been successful.

First, it is possible that the association between a diet rich in fruits and vegetables and the decreased risk of several diseases is a function of lifestyle differences; one or more factors - such as smoking, alcohol consumption, supplement use - may actually be responsible for the risk of some diseases. This hypothesis is considered unlikely by most nutrition scientists, given the repeatability of the inverse associations between fruit and 


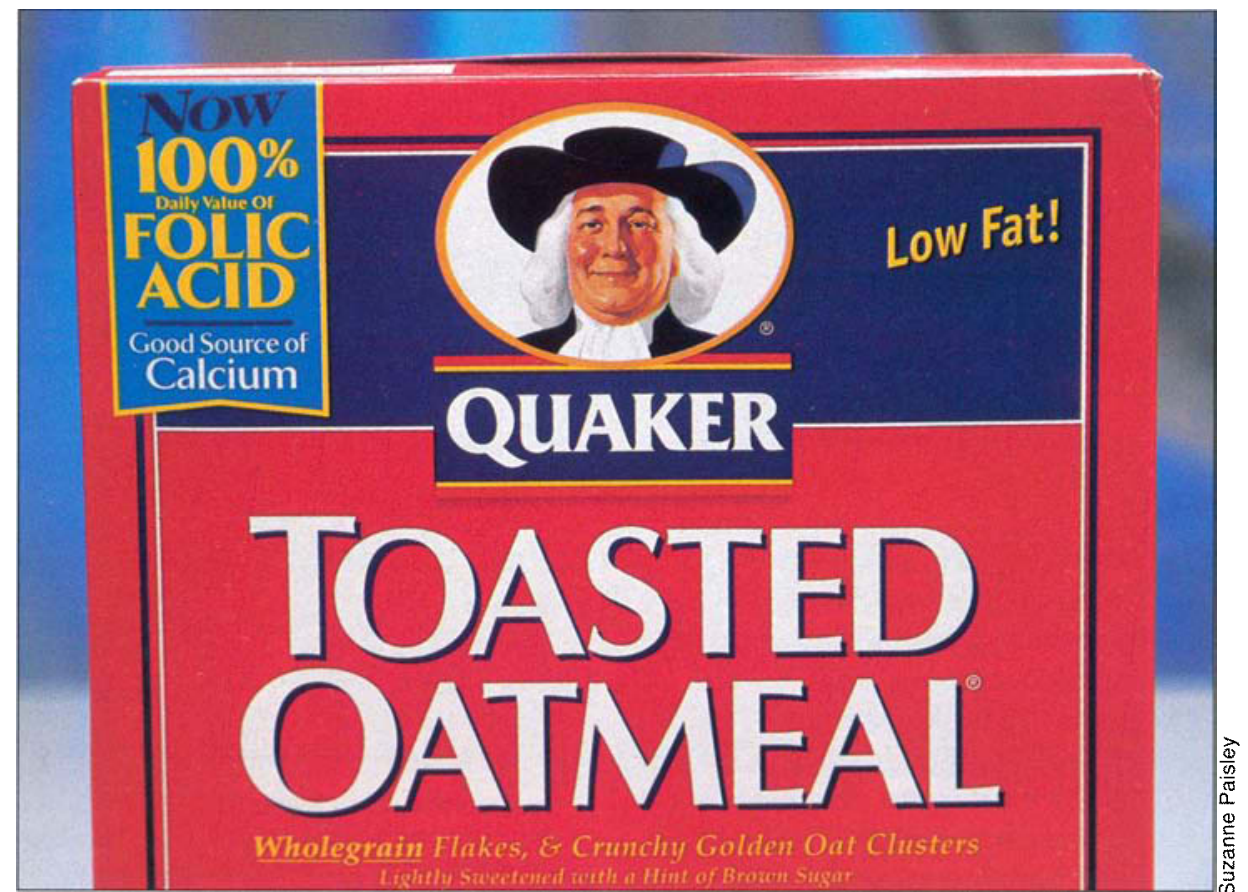

Between $68 \%$ and $87 \%$ of women of child-bearing age don't get the recommended levels of folate in their diets. Fortified foods, above, and dietary supplements can help to increase levels.

vegetable consumption and cardiovascular disease that have been observed, and the extensive use of covariate analysis, which in theory should identify these other factors.

Second, the health-promoting effects of fruits and vegetables may be attributable to other food components, such as phytochemicals that we are just beginning to study in-depth. For example, epicatechins, anthocyanidins, phytoestrogens, sterols and glucosinolates have all been reported to contribute to the health benefits of some foods (Piironen et al. 2000; Cassidy et al. 2000; Mithen et al. 2000; Clifford 2000; Rein et al. 2000).

Third, the long-term protective effects associated with certain phytochemicals may only be expressed when mixtures of these compounds (such as those found in whole foods) are consumed. This concept is supported by the observation that the antioxidant effects of vitamins $\mathrm{C}$ and $\mathrm{E}$ in human plasma are prolonged in the presence of the phytochemical catechin, which is found in many foods including numerous fruits and vegetables, green tea, red wine and chocolate (Lotito and Fraga 1998).

These possibilities underscore the importance of gaining a better under- standing of the metabolic actions of the phytochemicals present in our foods and how they interact. It will be important to maintain, indeed increase, our research efforts in this area during the next decade. Fruits and vegetables can vary tremendously in their concentrations of many of the putative protectant phytochemicals that are currently being studied. This can be due to a number of factors including the genetic background of the crop, local soil, climate, food processing and storage conditions. All fruits and vegetables are not equal! While considerable progress has been made in the study of food phytochemicals, we still have a long way to go during the next decade. However, we envision that identifying the compounds that confer protection, and at which concentrations, will form the rational basis for evaluating individual foods for their ability to influence the risk for particular diseases.

\section{Role of agricultural industry}

Presumably, with the identification of protective compounds, the agricultural industry can develop approaches aimed at the enhancement of these nutrients in commercially important crops. In theory, the concentrations of many of these phytochemicals in crops can be altered through changes in agronomic practices, as well as through traditional crop-breeding techniques and genetic modifications. The concentrations of protectant phytochemicals can also be increased in some foods by changes in postharvest processing and storage. These approaches are already being used in many countries and are at the heart of the European Union's NEODIET Program (Lindsay and Clifford 2000).

While the genetic engineering technology to modify the nutrient content of most plants is already available, a reasonable question must be asked: Are such modifications really needed to improve the health of our population or do they simply reflect shortcuts to good diets? Rather than developing such foods - with all of the associated economic and political ramifications - can conditions of optimal nutritional status be met through the consumption of typical present-day diets, particularly if one follows patterns of food intake that are consistent with our current dietary guidelines?

If current "natural" diets do not provide the optimal levels of nutrient intake, the importance of work in this area is amplified and new questions arise. For example, if typical diets (even those following dietary guidelines) do not provide the amounts of protective nutrients needed for optimal health, should this trigger the initiation of new food fortification programs, the use of dietary supplements or the development of new methods and approaches for changing the nutrient content of particular foods?

Before addressing these politically - as well as scientifically - loaded questions, we consider whether individuals can get "all that they need" from a typical diet; in particular, the influence of a pregnant woman's diet on the outcome of her pregnancy.

\section{Influence of diet on pregnancy}

Birth defects occur in about $3 \%$ of all live-born infants and are the leading cause of death among children in the United States. Even with intensive 


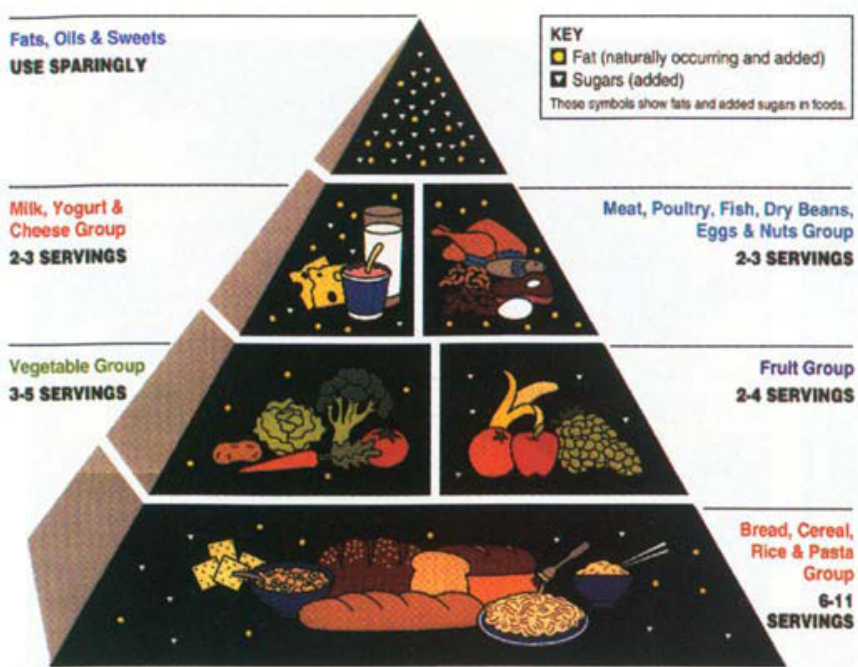

Consumption of fruits and vegetables, as recommended by the U.S. Food Pyramid Guide, above, may reduce the risk of heart disease, cancer and poor pregnancy outcome; however, the guidelines may not provide the recommended levels of several important nutrients, such as zinc and folate. A UC nutrition educator, right, demonstrates dietary portions.

investigation, causative factors can be identified for only $35 \%$ to $60 \%$ of the developmental defects reported in typical demographic populations, with specific teratogenic agents, monogenic defects and chromosomal defects accounting for $8 \%$ to $10 \%, 15 \%$ to $25 \%$ and $15 \%$ to $28 \%$ of the defects, respectively (Kimmel et al. 1993). We, and others, have argued that suboptimal maternal nutrition can be a significant factor underlying such pregnancy complications.

It has long been suspected that the quality of the maternal diet may have a significant influence on pregnancy outcome. In an early study by Ebbs et al. (1941), "poor" diet (defined as low in protein, calcium, fruits and vegetables) was correlated with a high incidence of miscarriages, stillbirths and early neonatal mortality compared to a "good" diet. Significantly, in a subset of women in the poor diet group who were given food supplements, the frequency of pregnancy complications was reduced to a level similar to that observed in the good diet group. This observation was critical as it suggested that the poor pregnancy outcome observed in the poor diet group was primarily due to nutrition per se, rather than other lifestyle factors.

Similar observations of an association between poor diet and poor preg- nancy outcome have been made by numerous research groups, as has the observation that improvements in pregnancy outcome in poor diet groups can be accomplished through the provision of dietary and food supplements (Keen 1996; Keen et al. 1997). Complementing these studies, numerous investigators have reported that the use of vitamin/mineral supplements during the periconceptional (preconception) period has reduced the predicted frequency of birth defects in certain populations (Keen et al. 1997; Berry et al. 1999; Czeizel 1996).

Single vs. multinutrients. Some investigators have argued that the reproductive benefits associated with the use of multivitamin/mineral supplements can, to a significant extent, be attributed to their folic acid. content. However, several micronutrient deficiencies may also contribute to the occurrence of birth defects (table 1). It is unlikely that the difference between "good" and "poor" diets with respect to pregnancy outcome can be attributed to a single nutrient.

While the use of periconceptional vitamin supplements (particularly folic acid) is advocated by many health agencies, including the U.S. Centers for Disease Control and Prevention (CDC), some controversy surrounds this issue. One argument against advocating the use of nutrient supplements is that some pregnant women may assume that the use of a supplement will by itself ensure that their nutritional status is adequate, losing their motivation to make healthy diet decisions. While this argument is commonly advanced, data supporting it have yet to be published.

Over- and undersupplementation. A second argument is that few studies demonstrate a link between primary

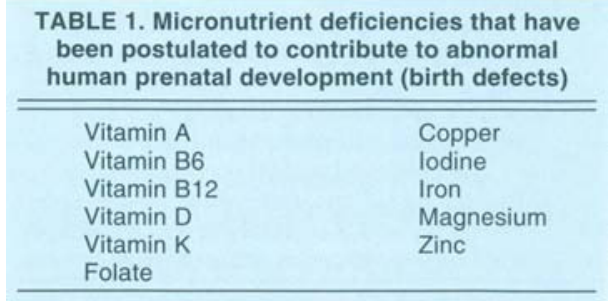




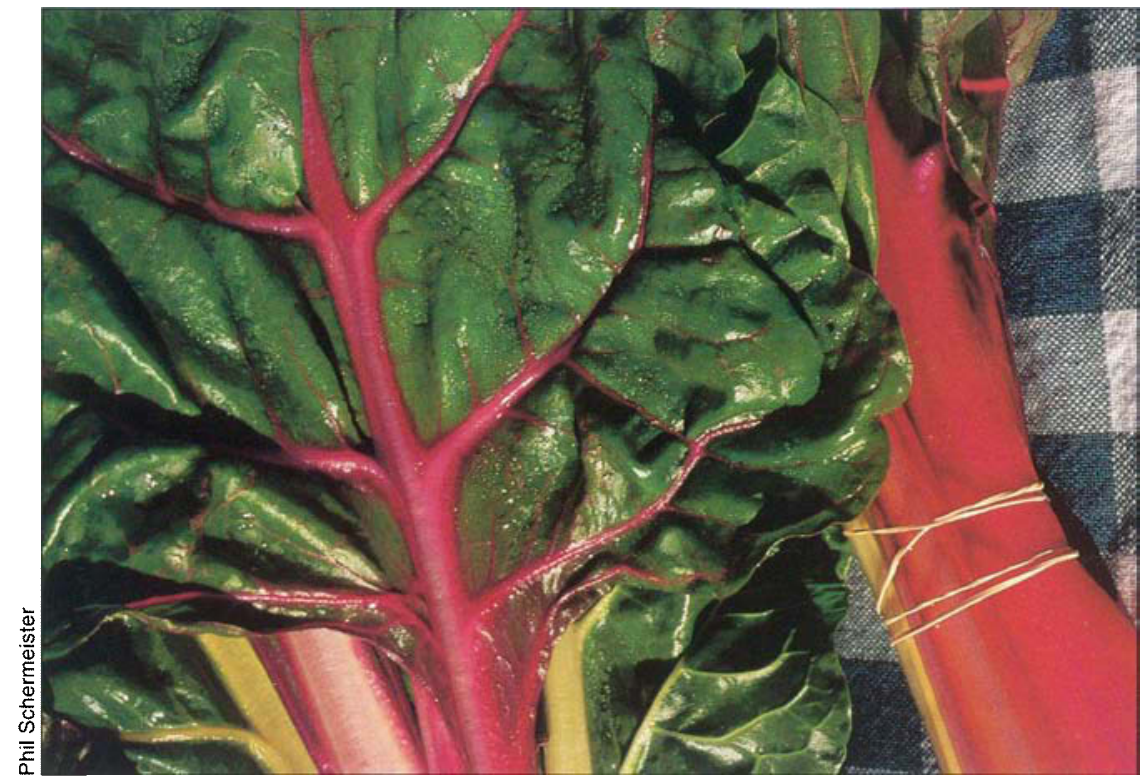

Not all fruits and vegetables are created equal. Concentrations of important nutrients can vary tremendously by crop, soil and climate conditions, food processing and storage method.

TABLE 2. Proportion of women aged $19-50$ years with intakes below $70 \%$ of the 1989 RDA on 4 nonconsecutive days

\begin{tabular}{lcc}
\hline \hline Nutrient & RDA & $<70 \%$ RDA \\
\hline & & $\%$ \\
Vitamin A & $800 \mathrm{RE}$ & 54 \\
Vitamin B6 & $1.6 \mathrm{mg}$ & 62 \\
Folate & $180 \mu \mathrm{g}$ & 36 \\
Calcium & $800 \mathrm{mg}$ & 60 \\
Iron & $15 \mathrm{mg}$ & 70 \\
Zinc & $12 \mathrm{mg}$ & 57 \\
\hline
\end{tabular}

Data are from the US Department of Agriculture (Human Nutrition Information Service 1988). The values are for nonpregnant women with family incomes $<131 \%$ poverty. Intakes were calculated from food frequency questionnaires collected on 4 nonconsecutive days. The RDA for calcium is 1,200 $\mathrm{mg}$ for women aged $19-24$, and $800 \mathrm{mg}$ for women aged 25-50. The $800 \mathrm{mg}$ RDA was used for the calculation above.

TABLE 3. Causative factors in nutrient deficiencies

Primary deficiency-low dietary intake of the nutrient Secondary ("conditioned") deficiency Genetic factors Mutant genes

Polymorphisms

Nutritional interactions

Dietary binding factors (fiber; phytate)

Nutrient-nutrient interactions ( $\mathrm{Zn}-\mathrm{Cu} ; \mathrm{Fe}-\mathrm{Mn}$ )

Disease-associated changes in nutrient metabolism

Drugs or other chemicals/toxicants

Direct metal chelation (decreased absorption/increased excretion)

Decreased gut absorption and/or increased kidney loss (secondary to tissue damage)

Toxicant induced changes in tissue pools maternal dietary deficiency of a nutrient and specific developmental defects. Given the rarity of primary maternal nutritional deficiencies (such as those that occur as a result of very low dietary intakes of the nutrient), the risks associ-

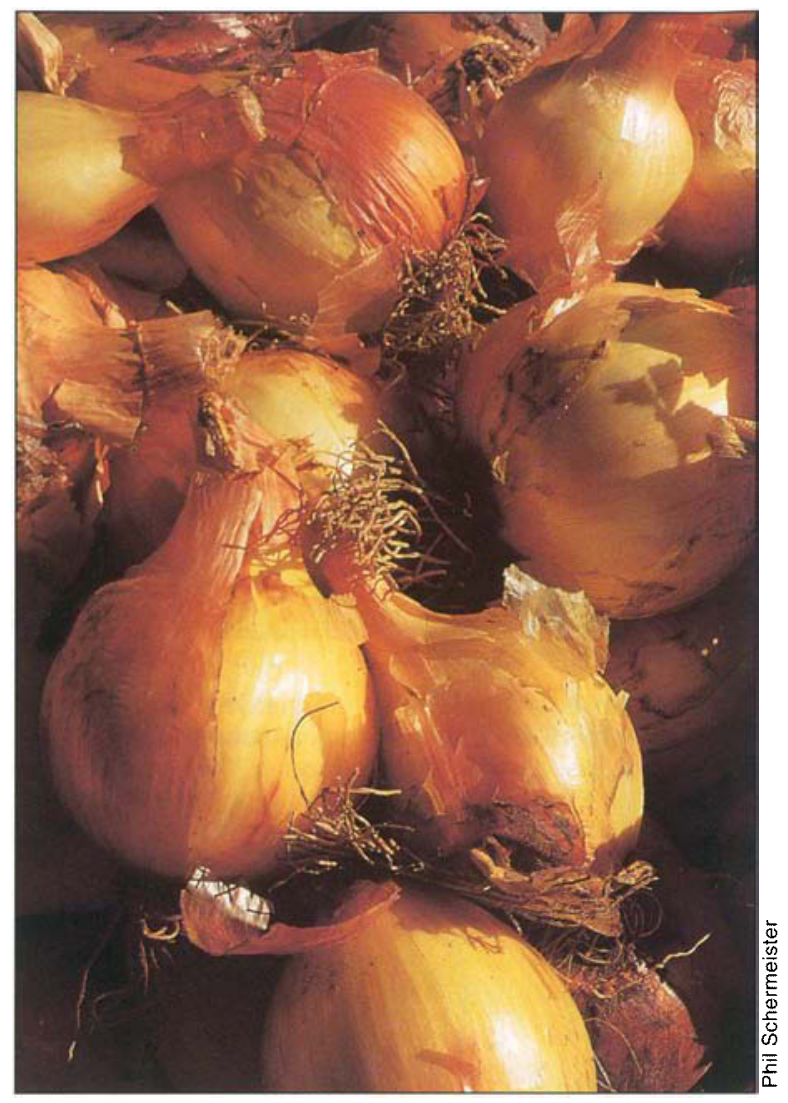
ated with the potential overuse of supplements might outweigh their benefits. However, a significant proportion of women of reproductive age are characterized by lower dietary intakes of select nutrients than currently recommended levels (table 2 ). Rather than being rare, marginal nutritional deficiencies in the United States may in fact be quite common. The influence of these marginal deficiencies on the developing fetus is not well understood; in addition to direct effects on the developing fetus, marginal nutritional deficiencies can also increase the risk for adverse reactions to other potential reproductive hazards. For example, in experimental animals, it is well recognized that the developmental toxicity of several diverse compounds can be amplified when the mother is fed a diet that is marginal in one or more nutrients (Keen et al. 1997).

Finally, our estimates of nutritional status may be in considerable error when based only on an inspection of dietary intakes, because an individual's nutritional status can be influenced by a number of other variables including genotype, environment, lifestyle habits, the presence of disease and drug/toxicant exposures (table 3).

\section{Toxicant-nutrient interactions}

Indeed, toxicant-nutrient interactions may underlie the occurrence of a significant proportion of human pregnancy complications. The increased breakdown of folic acid, which can occur via oxidative damage linked to smoking or alcohol consumption, as well as the accelerated breakdown of ascorbic acid observed in smokers, illustrate how certain toxicants can increase the metabolic need for particular nutrients (Scott et al. 2000; Lykkesfeldt et al. 2000). If this increased need is not met, the health of a developing fetus may be compromised.

Similarly, the developmental toxicity of numerous diverse toxicants including alcohol, cadmium and arsenic (Keen 1996) - in experimental animal models is due in part to their effects on maternal zinc metabolism. In essence, these compounds can trig- 
ger changes that reduce the delivery of zinc to the developing embryo/fetus. Significantly, the detrimental effects of these toxicants can be reduced when the mothers are fed diets high in zinc (Keen et al. 1997). Consistent with the findings from experimental animals, some investigators have reported that the frequency of pregnancy complications in human populations can be lowered with dietary folate and zinc supplements (Berry et al. 1999;

Goldenberg et al. 1995).

The levels of zinc and folate supplementation that have been reported as beneficial to pregnancy, typically exceed the amounts of these nutrients one typically gets even from "good diets." For example, diets following the Department of Agriculture's Food Guide Pyramid may provide only about half of the current recommendation for zinc and folate (Keen and Zidenberg-Cherr 1994). The above calculations were based on diets consumed prior to fortification of grains. However, recent reports suggest that $68 \%$ to $87 \%$ of females of child-bearing age still consume less than the recommended intake of 400 micrograms folate (Lewis et al. 1999).

With respect to the question "Can individuals get all that they need from a typical diet?" the answer is no, if the objective is to optimize pregnancy outcome. We suggest that similar arguments can be made for other nutrients, and other stages of life. For example, both children and lactating women can have difficulty in meeting their calcium requirements, particularly if they are lactose intolerant. With respect to zinc and folate, we recognize that dietary levels can be increased via fortification programs or the use of supplements. However, in our opinion, a more viable and palatable approach is the development of new plants that have an increased content, or bioavailability, of these nutrients (Scott et. al. 2000; Frossard et al. 2000).

\section{Future in focus: Research needs}

Although an adequate intake of most nutrients can be obtained from a well-balanced, well-planned diet, such diets do not typically provide all nutrients at levels currently considered op-

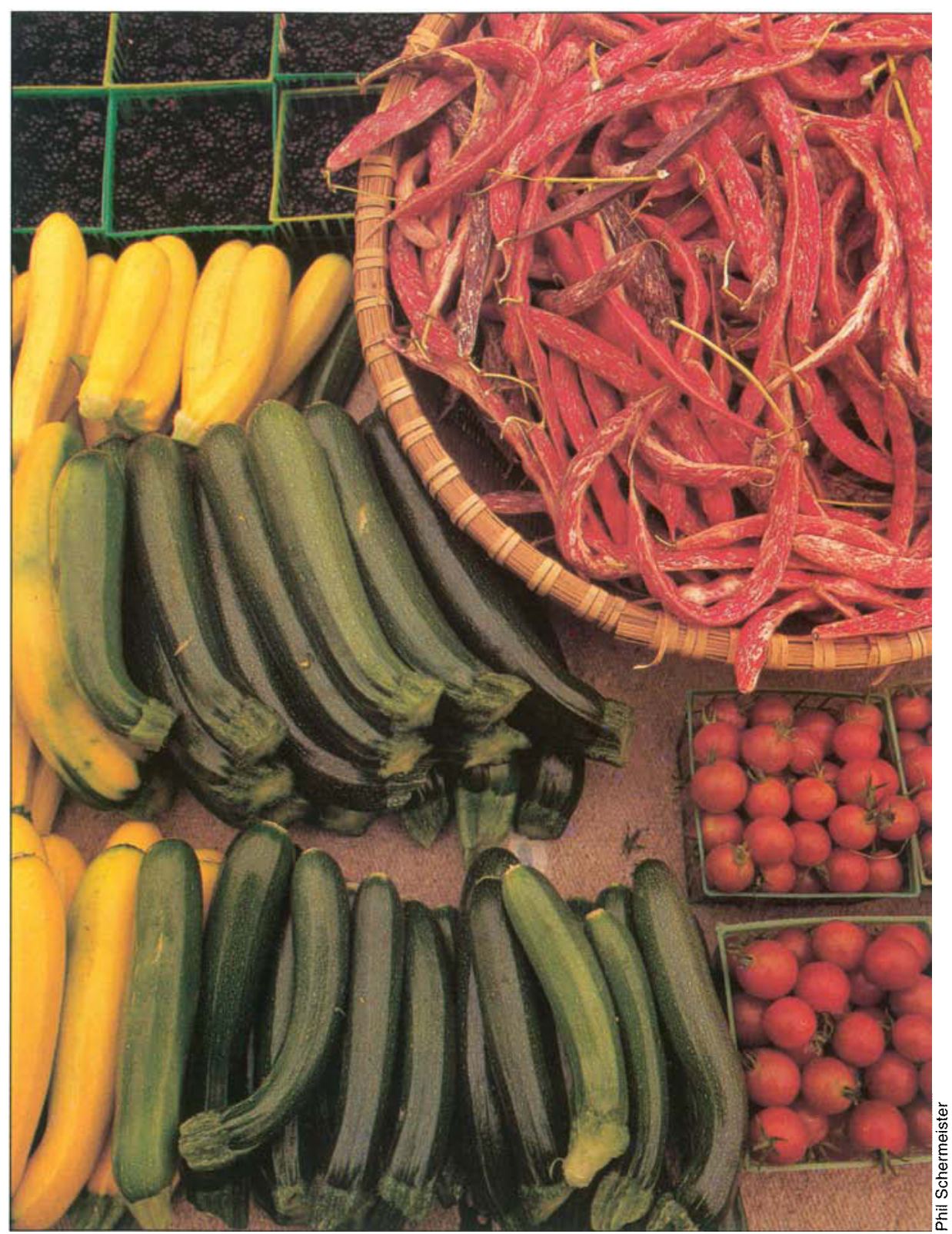

The nutrient composition of commonly consumed foods could be improvea tnrouy" conventional breeding techniques or genetic engineering. Supplemental nutrients may be needed for specific populations, such as pregnant women and the elderly.

timal by nutrition experts. Indeed, even when recent USDA food pyramid guidelines are used, it can be difficult to meet current recommended intakes for several essential nutrients such as vitamin $\mathrm{E}$, zinc and magnesium (Keen and Zidenberg-Cherr 1994). It is also possible that current recommended values for some nutrients are higher than necessary. The appropriateness of many of the recommendations is currently an area of vigorous research, and some values may be decreased in the future. However, it is equally possible that the current levels will be maintained, or even increased, for individuals with specific lifestyle habits, genetic backgrounds or chronic diseases.

If the current recommended values are correct, we must consider how to increase the population's intake of important nutrients. In the 21st century, the first step we will need to take is the development and implementation of targeted nutrition education programs, which provide the population with sound, science-based dietary recommendations (see p. 40). With respect to increasing the intake of select essential nutrients such as calcium, zinc, vitamin $\mathrm{E}$ and magnesium, one approach would be to increase their levels in target foods through fortifica- 


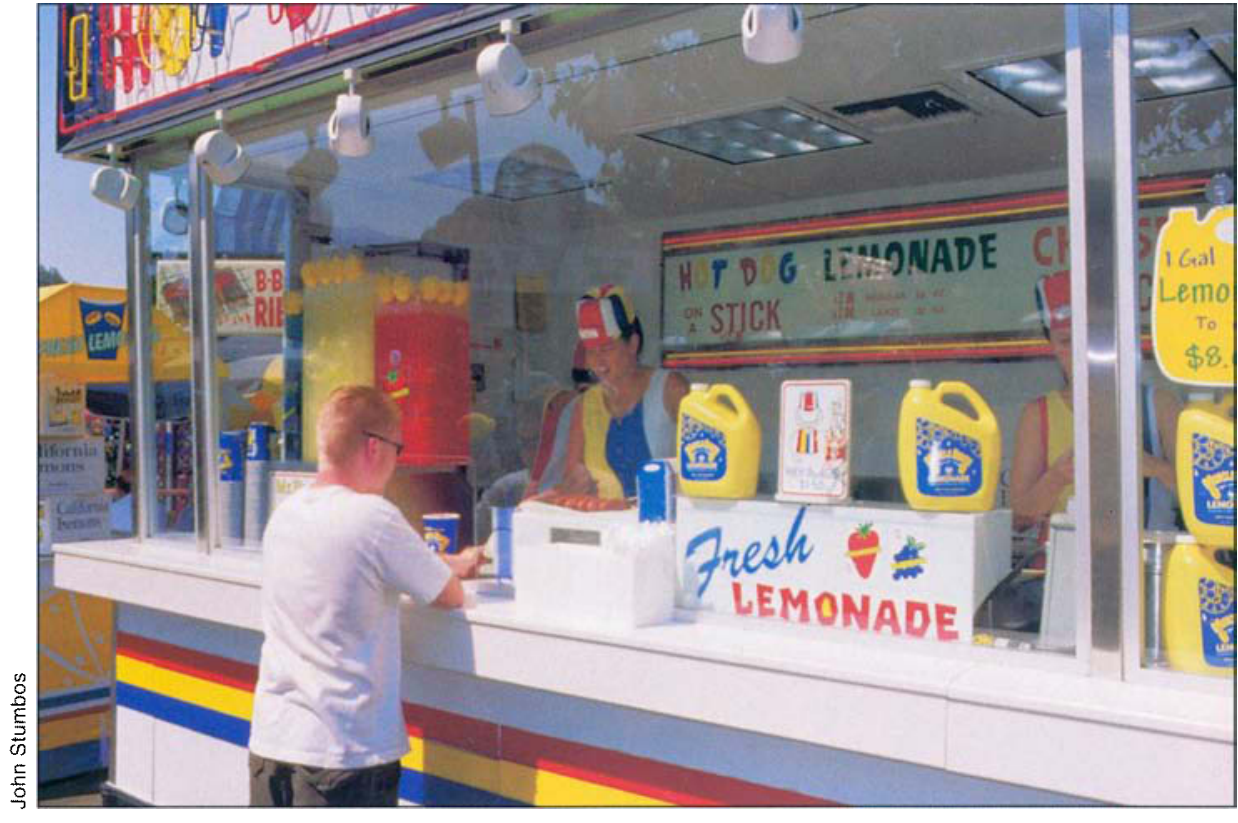

While "optimal nutrition" may be achievable in the future via food fortification and supplements, educational efforts to improve overall diets must continue.

tion. A second approach would be to change the nutrient composition of commonly consumed foods through changes in agricultural practices and through conventional breeding techniques or genetic modifications. A third approach would be to encourage certain populations such as pregnant women and the elderly to take daily supplements. If the third is accepted, the design of appropriate supplements should be a high priority for nutrition professionals.

Each of these approaches has its pros and cons, and we suggest that, for the near future, a combination of the three may be the best strategy. In addition, it is imperative for us to recognize that even with decades of investigation, we are just beginning to understand the complexity and diversity of health-promoting phytochemicals. We again emphasize the fact that even if new food fortification and supplement policies are promulgated, they should complement, not replace, educational and economic programs designed to improve the overall diet of the public.

C.L. Keen is Chair and Professor and S. Zidenberg-Cherr is Nutrition Science Specialist, Department of Nutrition, UC Davis.

\section{References}

[ATBC] Alpha-Tocopherol, Beta-Carotene Cancer Prevention Study Group. 1994. The effects of vitamin $E$ and beta-carotene on the incidence of lung cancer and other cancers in male smokers. New Engl J Med 330:1029-35.

Berry RJ, Li Z, Erikson JD, et al. 1999. Prevention of neural-tube defects with folic acid in China. N Engl $\mathrm{J}$ Med 341:1485-90.

Block G, Patterson B, Subar A. 1992 Fruit, vegetables and cancer prevention: $A$ review of the epidemiological evidence. Nutr Cancer 18:1-29.

Carr AC, Frei B. 1999. Toward a new recommended dietary allowance for vitamin $C$ based on antioxidant and health effects in humans. Am J Clin Nutr 69:1086-107.

Cassidy A, Hanley B, Lamuela-Raventos RM. 2000. Isoflavones, lignans and stilbenes - origins, metabolism and potential importance to human health. J Sci Food Agric 80:1044-62.

Clifford MN. 2000. Anthocyanins - nature, occurrence and dietary burden. J Sci Food Agric 80:1063-72

Czeizel AE. 1996. Reduction of urinary periconceptional multivitamin supplementation. Am J Med Genet 62:179-83.

Ebbs JH, Tisdall FF, Scott WA. 1941. The influence of prenatal diet on the mother and child. J Nutr 22:515-26.

Frossard E, Bucher M, Machler F, et al. 2000. Potential for increasing the content and bioavailability of $\mathrm{Fe}, \mathrm{Zn}$ and $\mathrm{Ca}$ in plants for human nutrition. J Sci Food Agric 80:861-79.

Goldenberg RL, Tamura T, Neggers $Y$, et al. 1995. The effect of zinc supplementation on pregnancy outcome. JAMA 274:463-8.

Hertog MGL, Kromhout D, Aravanis C, et al. 1995. Flavonoid intake and long-term risk of coronary heart disease and cancer in the Seven Countries study. Arch Intern Med 155:381-6. tract and cardiovascular defects by
[HOPE] Heart Outcomes Prevention Evaluation study investigators. 2000. Vitamin E supplementation and cardiovascular events in high-risk patients. N Engl J Med 2000 342:154-60.

Keen CL. 1996. Teratogenic effects of essential trace metals: Deficiencies and excesses. In: Chang LW, Magos L, Suzuki T (eds.). Toxicology of Metals. New York: CRC Lewis. p 977-1001.

Keen CL, Taubeneck MW, ZidenbergCherr S, et al. 1997. Toxicant exposure and trace-element metabolism in pregnancy. Env Toxicol and Pharmacol 4:301-8.

Keen CL, Zidenberg-Cherr S. 1994. Should vitamin-mineral supplements be recommended for all women with childbearing potential? Am J Clin Nutr 59:532S-9S.

Keli SO, Hertog MGL, Feskens EJ, et al. 1996. Dietary flavonoids, antioxidant vitamins and incidence of stroke: The Zutphen Study. Arch Intern Med 156:637-42.

Kimmel CA, Generoso WM, Thomas RD, et al. 1993. A new frontier in understanding the mechanisms of developmental abnormalities. Toxicol Appl Pharmacol 119:159-65.

Lewis CJ, Crane NT, Wilson DB, Yetley EA. 1999. Estimated folate intakes: Data updated to reflect food fortification, increased bioavailability and dietary supplement use. Am J Clin Nutr 70:198-207.

Lindsay DG, Clifford M. 2000. NEODIET Program; editorial. J Sci Food Agric 80:793-4.

Lotito SB, Fraga CG. 1998. (+)-Catechin prevents human plasma oxidation. Free Radic Biol Med 24:435-41.

Lykkesfeldt J, Christen S, Wallock LM, et al. 2000. Ascorbate is depleted by smoking and repleted by moderate supplementation: A study in male smokers and nonsmokers with matched dietary antioxidant intakes. Am J Clin Nutr 71:530-6.

Mithen RF, Dekker M, Verkerk R, et al. 2000 . The nutritional significance, biosynthesis and bioavailability of glucosinolates in human foods. J Sci Food Agric 80:967-84.

Pironen V, Lindsay DG, Miettinen TA, et al. 2000. Plant sterols: Biosynthesis, biological function and their importance to human nutrition. J Sci Food Agric 809:939-66.

Rein D, Paglieroni TG. Wun T, et al. 2000 Cocoa inhibits platelet activation and function. Am J Clin Nutr 72:30-5.

Scott J, Rébeillé F, Fletcher J. 2000. Folic acid and folates: the feasibility for nutritional enhancement in plant foods. J Sci Food Agric 80:795-824

US Department of Agriculture, Human Nutrition Information Service 1988. Nationwide Food Consumption Survey of Food Intake by Individuals. Women 19-50 and their children $1-5$ years, 4 Days. NFCS. Washington, DC. CSF11. p 86-3.

Yates AA. 1998. Process and development of dietary reference intakes: Basis, need and application of recommended dietary allowances. Nutrition Reviews 56:S5-9.

Yochum L, Kushi LH, Meyer K, et al. 1999. Dietary flavonoid intake and risk of cardiovascular disease in postmenopausal women. Am J Epidemiol 149:943-9. 